INTERNATIONAL DESIGN CONFERENCE - DESIGN 2018

https://doi.org/10.21278/idc.2018.0407

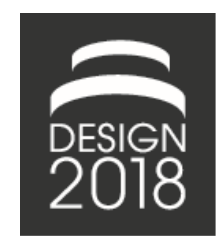

\title{
SUPPORTING TAILORING OF COMPLEX PRODUCT DEVELOPMENT PROCESSES: AN APPROACH BASED ON STRUCTURAL MODELLING AND ANALYSIS
}

\author{
C. Hollauer, M. Langner and U. Lindemann
}

\begin{abstract}
Project tailoring is discussed in academia and industry as a way to bridge the project-specific adaptation of generic organizational reference process models. Research into process tailoring in interdisciplinary product development is currently limited. We have developed a structure-based approach for supporting process tailoring, addressing limitations in existing approaches through: Concrete method support; systematic analysis of documented tailoring knowledge using structural analysis; and social aspects, e.g. stakeholder inclusion. The approach has been evaluated in eight case studies.
\end{abstract}

Keywords: design process, project management, process modelling, process tailoring, process adaptation

\section{Introduction and problem description}

Organizational standard models of product development processes (PDP) are considered a best practice with measurable positive impact on project performance, but in practice often considered too inflexible, with their creation also lacking a discernible methodology (Markham and Lee, 2013; Hollauer et al., 2016). Furthermore, support for creating design process models is currently limited aside from the use of specific formalisms, such as e.g. Gantt Charts or modelling notations (Gericke et al., 2016). Organizational PDP reference are often consciously kept ambiguous and vague, e.g. for audits, to hide the resulting variance caused by differences of individual project instances, reducing the usefulness of the model (Browning et al., 2006). Because designers and engineers are often forced to adhere to processes that do not fit their work reality, the developed process models can cause cognitive dissonance, resulting in frustration and their abandonment (Browning et al., 2006).

To contribute to bridging the gap between generic standard reference processes and project-specific instantiations, which arises due to different project objectives and boundary conditions requiring corresponding adaptations of the standard process, the concept of process tailoring has been discussed by a number of authors in literature, with respective approaches being developed (cf. Ginsberg and Quinn, 1995; Martinez-Ruiz et al., 2012; Graviss et al., 2016). Tailoring within the scope of this work is interpreted as the adaptation of organization-specific reference process models to the requirements posed by project boundary conditions via defined and documented guidelines or rules. This interpretation is rooted in the definition by (Ginsberg and Quinn, 1995), where tailoring is defined as "the act of adjusting the definitions and/or particularizing the terms of a general description to derive a description applicable to an alternate (less general) environment)". Consequently, explicit process tailoring has the potential to increase quality (in terms of comparability), transparency, traceability, efficiency (by reducing the required time), as well as learning within project planning through the 
documentation of recurring decisions. The resulting project-specific plans should be more suitable for the project situation at hand than simply reusing a standard process and thus can reduce the aforementioned cognitive dissonance. In order to successfully tailor PDPs, a thorough understanding of the process' context is required (Gericke et al., 2013). Process tailoring in practice is currently mostly applied implicitly and in an ad-hoc manner (Pedreira et al., 2007; Xu and Ramesh, 2008).

This paper contributes towards increasing the applicability of process tailoring in interdisciplinary product development (iPD) by providing an approach supporting the tailoring of complex PDPs, by applying structural modelling and analysis to manage the complex dependencies within the process as well as between the process and its application context. The paper gives an overview over the approach, as well as the initial application evaluation. In our empirical studies, we identified a lack of systematic tailoring application in iPD (used here synonymously for engineering design and systems engineering). Therefore, our work further contributes to closing this research gap by using a case study-based approach. We have further identified several research gaps within the existing approaches regarding systematic tailoring in literature (cf. Section 3.2).

The remainder of this paper is structured as follows: First, we present our research methodology after which the most relevant related work is briefly characterized. Subsequently, we present the developed design support, highlighting key aspects, as well as the initial evaluation of the design support, based on one case with two different processes, a PDP and one of its sub-processes, selected from our portfolio of case studies.

\section{Research methodology}

The performed research is motivated by the following research question: "What activities and supporting methods are necessary to support the tailoring of complex PDPs in iPD, with specific focus on organizational and social aspects?".

The research follows the Design Research Methodology (DRM) (Blessing and Chakrabarti, 2009), using case studies in (to date) six different organizations with varied boundary conditions as main empirical research method (cf. Table 1) (Yin, 2014). The case studies are numbered using a capital letter (company), as we all as a number (unit of analysis, i.e. a specific process or sub-process).

Table 1. Overview over conducted and ongoing evaluation case studies

\begin{tabular}{|c|c|c|c|c|c|c|c|c|c|}
\hline & Case & \multicolumn{2}{|c|}{ A } & $\mathrm{B}$ & \multicolumn{2}{|c|}{ C } & $\mathrm{D}$ & $\mathrm{E}$ & $\mathrm{F}$ \\
\hline \multirow{4}{*}{ 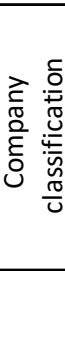 } & Size & \multicolumn{2}{|c|}{ large } & large & \multicolumn{2}{|c|}{ large } & medium & small & medium \\
\hline & Industry & \multicolumn{2}{|c|}{ Automotive } & Automotive & \multicolumn{2}{|c|}{ Trucks } & $\begin{array}{c}\text { Plant } \\
\text { engineering }\end{array}$ & $\begin{array}{l}\text { Medical and } \\
\text { Lab devices }\end{array}$ & $\begin{array}{c}\text { Production plant } \\
\text { engineering }\end{array}$ \\
\hline & Market & \multicolumn{2}{|c|}{$\mathrm{B} 2 \mathrm{C}$} & B2C & \multicolumn{2}{|c|}{$\begin{array}{c}\text { B2C/B2B } \\
\text { (OEM) }\end{array}$} & $\begin{array}{c}\text { B2B } \\
\text { (supplier) }\end{array}$ & B2B & $\mathrm{B} 2 \mathrm{~B}$ \\
\hline & $\begin{array}{l}\text { Unit of } \\
\text { Analysis }\end{array}$ & 1 & 2 & 1 & 1 & 2 & 1 & 1 & 1 \\
\hline \multirow{3}{*}{ 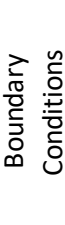 } & \begin{tabular}{|l} 
Process \\
Level
\end{tabular} & PDP* & $\begin{array}{c}\mathrm{SP} \\
\text { (Release) } \\
\end{array}$ & $\begin{array}{c}\text { SP } \\
\text { (Styling) }\end{array}$ & $\begin{array}{c}\mathrm{SP} \\
(\mathrm{P} \& \mathrm{~L})\end{array}$ & PDP & PDP & PDP & PDP \\
\hline & $\begin{array}{l}\text { Established } \\
\text { process }\end{array}$ & Yes & Yes & Yes & Yes & Yes & Yes & No & No \\
\hline & \begin{tabular}{|l} 
Tailoring- \\
Experience \\
\end{tabular} & no & no & no & limited & limited & no & no & no \\
\hline & $\begin{array}{l}\mathrm{P} \& \mathrm{~L}=\text { Deve } \\
\mathrm{PDP}=\text { Produ } \\
\mathrm{SP}=\text { Subpro } \\
*=\text { The PDP }\end{array}$ & $\begin{array}{l}\text { nent of } \\
\text { levelop } \\
\text { s withir } \\
\text { a spec }\end{array}$ & $\begin{array}{l}\text { oduction a } \\
\text { nt process } \\
\text { e PDP; tab } \\
\text { departmen }\end{array}$ & $\begin{array}{l}\text { d Logistics } \\
\text { (top-level) } \\
\text { e only show } \\
\text { t has been ir }\end{array}$ & $\begin{array}{l}\text { oncepts } \\
\text { currently } \\
\text { estigate }\end{array}$ & $\begin{array}{l}\text { finished } \\
\text { (Engine }\end{array}$ & $\begin{array}{l}\text { or finishing } \\
\text { control units }\end{array}$ & $\begin{array}{l}\text { ase studies } \\
\text { ) }\end{array}$ & \\
\hline
\end{tabular}

A review and characterization of existing process tailoring approaches forms the theoretical basis of the research clarification and descriptive study I (DS I), from which issues and research gaps presented in Section 3.2 have been derived (cf. Hollauer and Lindemann, 2017). The case studies are used to: 1) 
empirically investigate the current state in industry (DS I), 2) iteratively refine the requirements and design support (prescriptive study, PS), and 3) evaluate the design support and derive further improvement based on lessons learned during the application (descriptive study II, DS II). The design support has been iteratively advanced based on lessons learned from the application: While the scope and objective of the developed approach remained stable over its development, the design of the individual phases and specific method support have been iteratively extended and refined.

The evaluation presented in the paper at hand (Section 5) focuses on case studies C-1 and C-2, as both case studies have been conducted within the same company, but on different hierarchical process levels. Both case studies focus on the application and evaluation of phases 1-3: Acquiring information and building a TSM. During DS-II, the methodology was carried out by embedded students within the case studies, which were closely instructed in the application of the design support, regularly reporting their findings and observations. Regular meetings with expert stakeholders from the companies have been conducted in order to assess progress and feedback. Concluding evaluation interviews were held, were a summative evaluation of the applied elements of the design support was conducted.

\section{Background and related work}

\subsection{Terms and definitions}

The paper at hand presents an approach for process tailoring (cf. Section 1). Tailoring can be applied to different hierarchical process levels, in our case the PDP or a specific sub-process, e.g. a risk or requirements management process, a sub-process originating from a specific design department, or a sub-sub-process regarding a specific calculation within concept design. Within this paper, the term "tailoring rules" will be used. These rules serve as a notation to document reoccurring tailoring decisions for reusability and analysis. They consist of conditions (context factors) as well as impacts on process elements to be considered during process tailoring (cf. Figure 1). Context factors represent the variability within a processes application context (i.e. the characteristics of a company's project portfolio) and consist of a variable and corresponding values, a notation commonly used in existing tailoring approaches (e.g. Hurtado Alegria, 2012), and derived from feature modelling/domain engineering (cf. Czarnecki and Eisenecker, 2005). Examples for possible context factors and values are e.g.: The development task (new development, redesign, or variant development), a particular product variant (e.g. coupé or convertible), specific customers or aggregated customer groups (e.g. regular vs. strategically important customers), the fact whether a project includes external development partners, or whether a project concerns the development of a complete system, a module, or a single component.

\subsection{Process tailoring: Related work and research gaps}

Regarding related tailoring approaches, a short characterization of existing approaches will be given within this paper, with the intention to highlight the identified research gaps. A detailed overview over existing approaches is not focus of the paper at hand (cf. Hollauer and Lindemann, 2017; or MartinezRuiz et al., 2012; for existing reviews).

Process tailoring approaches employ different means of documenting and storing tailoring knowledge: Case (i.e. identifying old project plans based on project characteristics), model, and rule-based approaches (cf. Helms, 2013). Kang et al. (2008) is one example for a case-based approach. The latter two classes are often being used in combination (e.g. Hurtado Alegria, 2012). We focus on model and rule-based approaches, since they are better suited to document expert knowledge (Helms, 2013). In our literature review, we have selected 14 relevant approaches that fit our inclusion criteria.

The majority of identified approaches originates within the field of software engineering, with related work in fields such as Situational Method Engineering (Henderson-Sellers and Ralyté, 2010). However, no unified approach or industry standard is evident. Based on our review we identified the following research gaps within existing approaches:

- Within the domain of iPD, research regarding empirical descriptive studies as well as prescriptive approaches is still limited (e.g. Graviss et al., 2016), while the need for process 
contextualization/tailoring is generally acknowledged in literature (e.g. Haskins, 2011; Gericke et al., 2013; Andreasen et al., 2015; Walden et al., 2015; Bender and Gericke, 2016).

- Existing approaches are either very generic, addressing only the basic principles of tailoring and steps that should be taken without elaborating them (e.g. Zakaria et al., 2015; Graviss et al., 2016), or very specific, being only applicable in certain domains, processes, or requiring specific technologies to automate the tailoring activity (e.g. Hurtado Alegría et al., 2011).

- Existing approaches largely focus on the automation of the tailoring activity itself, using complex modelling approaches requiring domain-specific software skills and knowledge, and depend on particular technical implementations (i.e. software systems, e.g. Hurtado Alegria, 2012; using the Atlas Transformation Language).

- Existing meta models for the documentation of tailoring knowledge are often extensions of specific process modelling languages, providing a strong basis but limiting their general applicability (e.g. SPEM, cf. Hurtado Alegria, 2012; or BPMN, Pillat et al., 2015).

- The acquisition of organization-specific context factors and the methods to do so are either not described in detail, or generic context factors are used.

- Social and organizational aspects are so far underrepresented in existing approaches. Since process tailoring is knowledge-intensive and complex (Xu, 2005), it requires intensive communication, coordination and expert judgment in preparing as well as executing the tailoring activity.

- Means for a systematic analysis of the acquired tailoring knowledge (e.g. context factors or rules) are not provided, e.g. for the systematic classification of tailoring rules and derivation of a tailoring process.

To summarize, we identified the need for an approach that supports a structured, systematic process tailoring in $\mathrm{PDD}$, by providing operative guidance, a pragmatic modelling approach, and engaging stakeholders in the tailoring activity in order to foster communication regarding tailoring decisions. We decided to base the approach on structural modeling and analysis (cf. Section 3.3) of complex processes to improve the preparation as well as application of process tailoring, instead of implementing and automating the knowledge regarding tailoring decisions in a software system which represents a "black box" for process stakeholders. Workshops with relevant project stakeholders are subsequently used as the main means to coordinate, discuss and apply the process tailoring decisions.

\subsection{Structural modelling and analysis}

The tailoring-relevant knowledge as described in Section 3.1 represents a structure. To document the tailoring-relevant knowledge, we make use of multi-domain graph- and matrix-based modelling approaches (in this paper summarily called structural models). Structural models are used to document and analyse elements (nodes) and their dependencies (edges) across single (e.g. design structure matrix, DSM) or multiple domains (e.g. domain mapping matrix, DMM or multiple-domain matrix, MDM) (cf. Maurer, 2007). Such models have been previously used to successfully model as well as analyse complex PDPs (Kreimeyer, 2010). As graphs and matrices can be transferred into each other, both are equivalent representations of the same system (Tittmann, 2003, p. 29). To analyse structural models, a variety of options is available:

- Structural metrics, representing indicators to identify and characterize particular patterns of interest, e.g. the significance of nodes in terms of their centrality or the distance between two particular nodes. Metrics can be categorized into basic metrics (e.g. number of elements of a domain) and more elaborate, combined metrics (Kreimeyer, 2010; cf. Maurer, 2007).

- Pattern search, where patterns of interest are matched within the investigated model (primarily graph-based). This approach can be used in order to calculate the aforementioned structural metrics (Kissel, 2014).

- Algorithms for clustering and sequencing (Browning, 2001) (primarily for single-domain models and matrix-based representations, such as DSMs), where matrices are reorganized in order to e.g. derive groups nodes with high connectivity or an optimal sequence of nodes. 


\section{Support for implementing process tailoring in complex PDPs}

\subsection{Overview and general structure}

The proposed approach consists of two main elements: A meta model (Figure 1) defines the language to document the tailoring-relevant knowledge as a "TailoringSystemModel" (TSM) and an accompanying structural analysis framework provides several means for analysing the TSMs structure (e.g. structural metrics). Structural analyses are then applied to the TSM to derive insights regarding information on how the tailoring is to be carried out, e.g. the sequence of rule application (triangularization of dependencies between context factors), the workshop-structure and stakeholders to be involved per workshop (common tailoring concerns due to common process elements and rules between stakeholders), and the characterization of tailoring rules (e.g. due to number and criticality of impacted process elements). An overarching methodology structures the sequence of activities to be performed and provides method support regarding individual activities, i.e. the acquisition of information to create the TSM (cf. Figure 2). The subsequent sections focus on presenting the modeling framework and methodology, while the analysis framework is only outlined briefly.

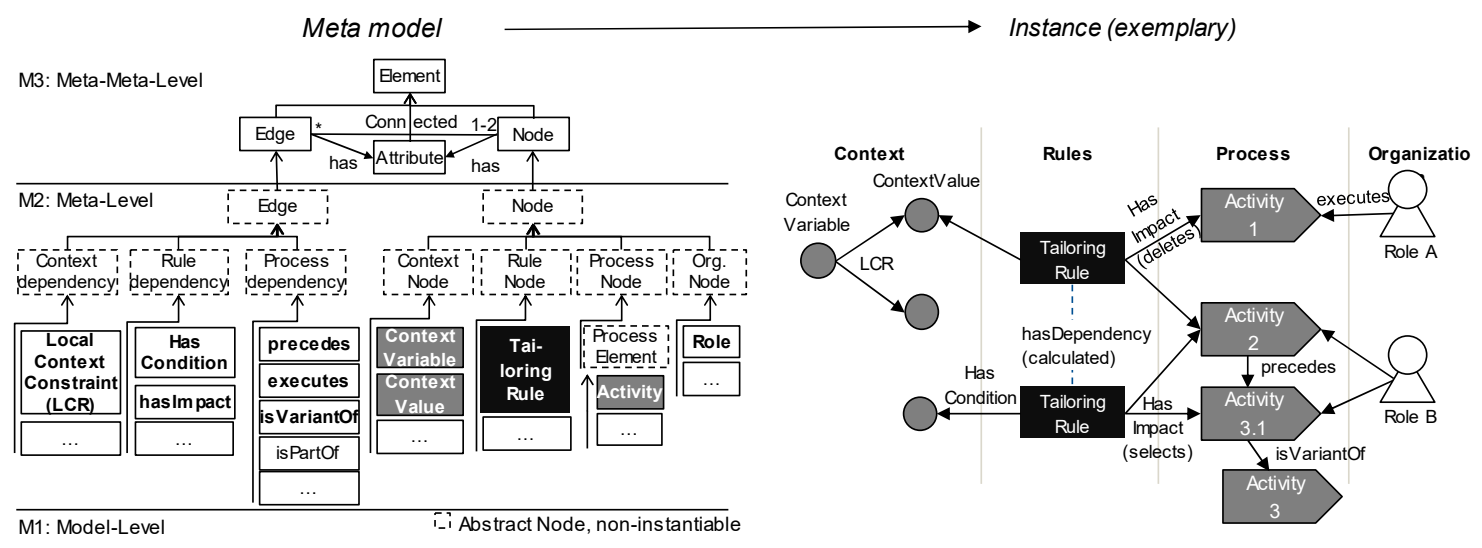

Figure 1. Meta model for TailoringSystemModel showing element inheritance (left, abbreviated) and example for an instance of tailoring rules and their constituent elements (right)

\subsection{Modelling framework: Meta model and example}

The meta model (cf. Figure 1) has been developed by reusing and adapting elements from existing meta models, e.g. Hurtado Alegria (2012) for process context and variability modelling, Park et al. (2006) for tailoring rules, Kreimeyer (2010) and Chucholowski and Lindemann (2015) for process modelling. In order to capture the relevant information, the meta model comprises the domains of context, rules, process, organization and captures dependencies within and between these domains (cf. Figure 1).

The meta model has been prototypically implemented and tested for applicability as a relational database (Microsoft Access) for data input, as well as a graph-based modelling tool to support model visualization and subsequent structural analysis (Soley, www.soley.io). While the meta model provides further constructs identified as relevant in case study D, these are not further elaborated here (e.g. constraint rules between context factors). For the individual elements of the meta model, specific attributes have been defined to support documentation.

\subsection{Methodology: Phases and supporting methods}

The overall phases of the developed methodology (cf. Figure 2) are derived from the structural complexity management approach by Lindemann et al. (2009). The basis of the design of the individual phases of the methodology was derived from existing model- and rule based process tailoring approaches in literature. 

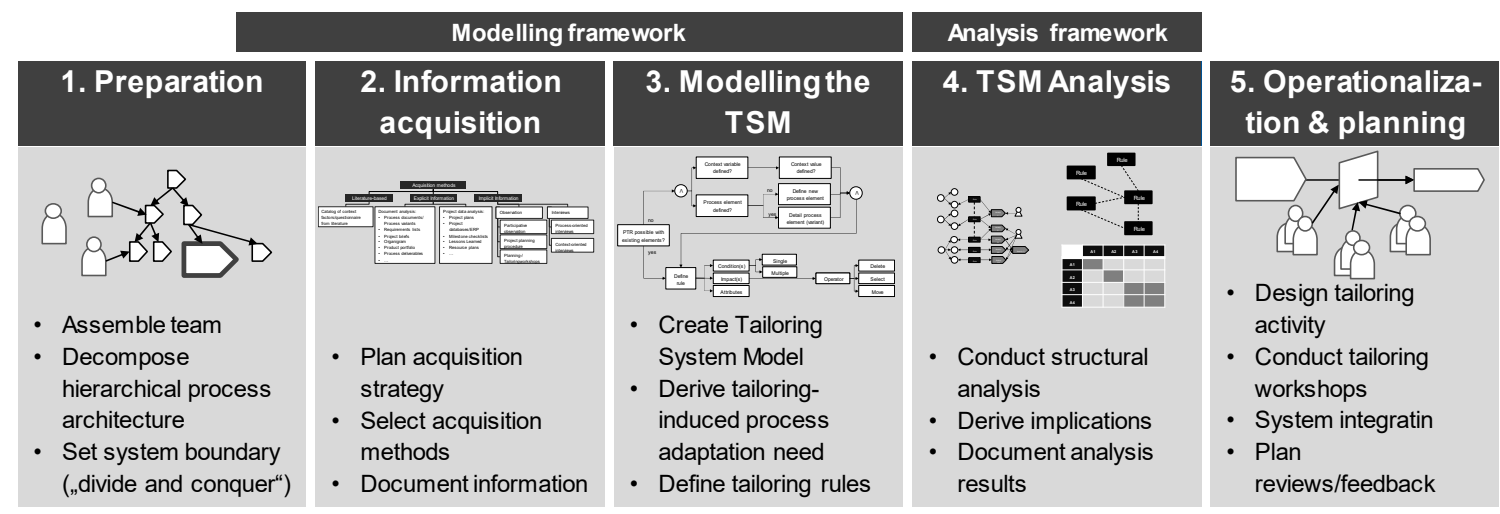

Figure 2. Overview of methodology: Phase structure and outline of individual phases

The methodology can be applied flexibly, by: 1) Extending the tailoring coverage to neighbouring subprocesses within the superordinate process (parallelization), 2) escalating the coverage to superordinate processes, or 3) further focusing and detailing particular sub-processes within already investigated (sub)processes (recursion). Since the motivation to create a tailorable process might differ between departments or sub-processes, e.g. due to different degrees of process variance, different starting points can be selected, subsequently feeding the information into a common TSM (cf. Section 5.3). This relates to the complexity management principle of "divide and conquer", since the application to a large process can be too complex and resource-intensive, reducing the chances of success.

The methodology itself is structured into five phases. During the preparation, the team responsible for the application of the design support is assembled. To support this, different roles have defined. For example, the role of one or more "tailoring expert" should be implemented within the organization and filled by experts with both process and project experience (e.g. someone from a central project management office). As the methodology can be applied to different process levels (i.e. the PDP in its entirety or individual, particular sub-processes), another preparatory step is the analysis of the prevalent hierarchical process levels and selection of one or more system boundaries for application of the methodology. An initial stakeholder analysis within the chosen system boundary is then conducted to identify permanent as well as temporary members, e.g. process owners, project managers, engineers, and designers with extensive process knowledge for information acquisition. Method support within this phase are: hierarchical process modelling, stakeholder maps, or interface matrices between departments. A catalog of information acquisition methods supports the information acquisition phase (Figure 3 ). The methods have been developed by identifying existing methods regarding context acquisition in literature and subsequently contextualizing and concretizing these methods through repeated application in different case studies. Our literature review indicated that the existing methods for process context acquisition are: checklist-based acquisition, observation, and interviews (group and individual) (cf. Badke-Schaub and Frankenberger, 2004). The methods in the provided catalog are grouped according to the information sources used: Literature-based information (pre-established list of generic context factors), explicit, and implicit information within the organization. Literature-based information can serve as a starting point to get an overview, but is rarely sufficient as seen in our case studies, since for each organization and particular sub-process therein, different factors are relevant. The analysis of explicit, historical project data (e.g. schedule or budget plans) is a valuable source to identify process variance, given enough data in terms of quality and quantity (e.g. identifying the overall frequency with which activities are part of project plans or the significance with which activities occur together in different project plans, as applied in case study D-1). Implicit information can be accessed through: participative observation (requires a small enough system boundary to be feasible), the observation of project planning procedures as conducted by individual experts (project planners or managers), or a planning-/process tailoring workshop for a particular project involving multiple stakeholders. Interviews with individuals can be used to gain new information or to validate findings previously gained through explicit information or observations. Interviews are differentiated into process- and context-oriented, with the former targeting operative designers and developers investigating process variances and 
deriving underlying causes, and the latter aimed at management staff that is less familiar with detailed tasks but instead has more overview regarding possible influences on the process and their impact.

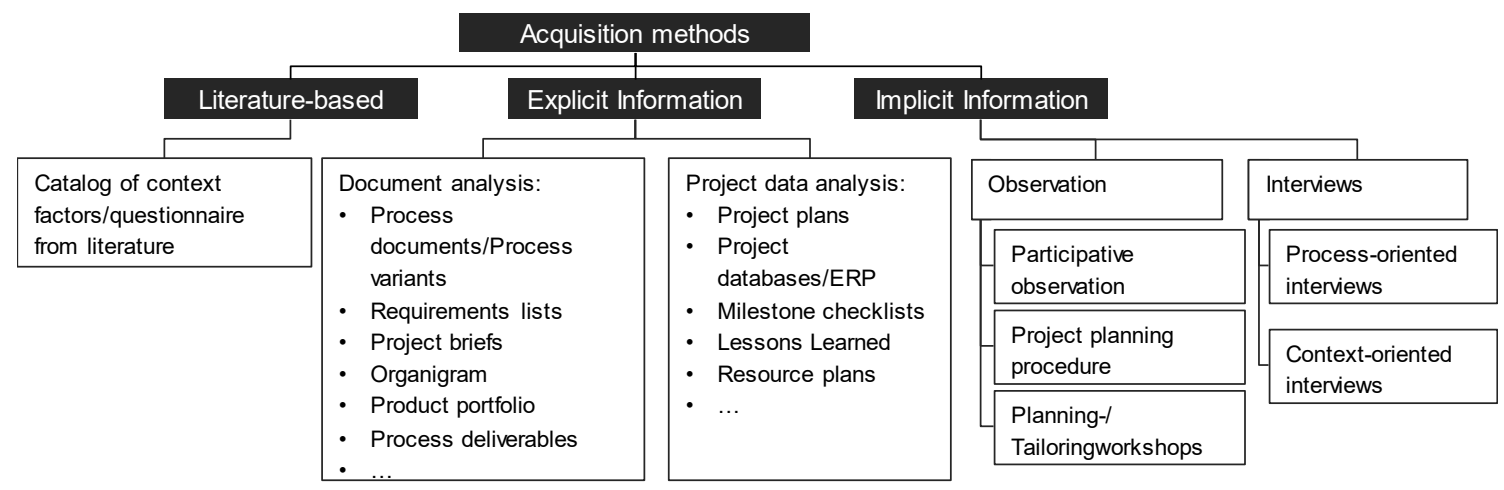

Figure 3. Methods for acquiring context factors and process variance

During the modelling phase, the meta model (Figure 1) is applied to create the TSM, with three objectives: 1) defining a comprehensive set of tailoring rules, 2) deriving the tailoring-induced need for process adaptation, and 3) documenting the tailoring information for subsequent analysis. As experiences during the case studies have shown, existing process models are not always directly suited for the definition of tailoring rules, especially when the tailoring impacts are derived on a needs basis from the process context (cf. Section 5.3). The methodology provides guidelines on how to handle different modelling situations: e.g. in case tailoring rules cannot be defined directly, because the identified activities are not defined in the process model, process adaptation operators are provided with which to adapt the process model to increase its tailorability. Another guideline prescribes the definition of tailoring operators for tailoring rules, which consist of either deleting an activity from the reference process model, selecting a specific activity mode (activity variant), or moving an activity to another position within the process.

Based on the TSM, structural analyses can then be conducted to derive implications for adapting the process to be tailored as well as the corresponding tailoring activity. For the analyses, the TSM is either analysed directly, or data is extracted from the TSM. Within this paper, we outline possible structural analysis, their elaboration and implementation being subject of current work:

- Modularizing the process for tailorability via indirect dependencies between process elements by context values, using a matrix-based clustering algorithm

- Analysing context configurations to derive most frequently required process variants for most commonly occurring context value combinations

- Classifying tailoring rules according to their complexity by using structural metrics, e.g: the number of conditions and impacts of a rule; the criticality, centrality, and the snowball factor of impacted process elements per rule, and the distance between impacted process elements as well as impacted stakeholders (spread of impacts over process and organizational hierarchy) (cf. Kreimeyer, 2010)

- Derivation of indirect dependencies between rules (e.g. due to possible conflicts of operations on the same activity)

- Triangularization of tailoring rules according to their indirect dependencies (via context or process elements) for derivation of an efficient sequence of execution

- Calculation of indirect stakeholder-rule relationships and their criticality to identify the importance of individual process stakeholders (e.g. roles) for tailoring

- Calculation of indirect stakeholder relationships via rules and matrix-based clustering to derive cohesive groups for stakeholder workshops

Operationalization \& planning addresses activities regarding the operationalization and subsequent maintenance and improvement of the tailoring activity within the organization. The information generated during the analysis phase is used to design and integrate the tailoring activity within project 
planning: Tailoring workshops are planned based on the identified communication needs and required supporting measures need to be prepared (e.g. a tailoring handbook containing the standard process as well as the ruleset, prioritized based on the calculated complexity and impact of the tailoring decisions). Subsequently, reviews of the TSM are required on a regular basis. These reviews depend, among other, on the frequency with which projects are started and concluded (generation of new data) and should be planned, using e.g. a roadmapping approach. To further support the review process as well as future implementation of technical solutions to support the tailoring activity, a system integration map can be used, highlighting information flows between technical systems (e.g. between project and process management systems).

\section{Evaluation case study: Commercial vehicle OEM}

Case study C (Table 1) investigates two units of analysis: The commercial vehicle OEMs overall PDP, approached from a high-level perspective of central project management (C-2), as well as the subprocess regarding the development of production and logistics concepts within design projects $(\mathrm{C}-1)$. The presented case study focusses on the feasibility and applicability of phases 1 to 3 of the design support, highlighting results from the information acquisition and general lessons learned during the application. The company has had some previous experience with process tailoring, but no formalized and structured tailoring process existed. Tailoring has been done in workshops, where the reference process is discussed along the activities with project managers from all department of a given project. No specific input besides the reference process model is used, nor is the knowledge specifically documented and reused. Not all required participants are always able to attend, are not required for the entirety of the workshop, nor do they always have the in-depth knowledge required for process tailoring.

\subsection{Unit of analysis 1: Sub-process "production \& logistics concepts"}

$\mathrm{C}-1$ focused on defining a tailoring ruleset for the department overseeing the development of production and logistics concepts for new truck launches. The department consists of several project managers supervising specialized project engineers responsible for the adaptation of existing production and logistics related processes when introducing a new design. Their activities include the design of new production and logistics concepts, construction and evaluation of prototypes, and material testing. The team works very closely with the design department and project managers report directly to the project leader.

Previously, the department itself had no established tailoring process nor any documented knowledge regarding context factors. In Phase 1, the levels of the process hierarchy were identified and the system boundary defined. The study further focused on the information acquisition (Phase 2) and the preliminary design of a practical tailoring process (Phase 5). To identify context factors, semi-structured interviews with project managers $(n=6)$ and project engineers $(n=7)$ were conducted. During interviews, the participants were asked to describe reasons why individual project plans deviated from the standard development process. Also, standardized milestone approval documents, used to document the steps taken to pass a formal milestone were analysed regarding reasons for not applying the standard process. Overall, 106 context factors with associated values were defined. For example, a commonly named factor (38,5\% of interview participants) was shell geometry: if the shell geometry of new parts does not change significantly, the prototyping necessary to ensure smooth assembly of the truck can be reduced drastically. Instead of various physical prototypes, a virtual prototype is sufficient to ensure assembly is possible. Moreover, transport containers and storage space can be reused. Another frequently mentioned context factor is that if production is switched to a new design from one truck to the next in one single change, no test series can be produced, eliminating activities related to the development of a test series as well as its usage as prototypes. For the rules defined based on these context factors, only the operator "delete" has been used, by request of the company. The rules were subsequently documented using spreadsheet software, with the modelling approach following the meta model presented in Section 0. Context variables, vales, as well as the affected process steps per value are documented. Besides this basic information, several other attributes like the timeframe of the effects of a factor were documented. Based on these findings and the needs of the department, an initial tailoring process was developed. The developed process addresses the integration of existing systems, which are currently used to manage the 
reference process as well as projects, by identifying the interactions between these systems. The case study is still ongoing, with current activities focused on adapting and detailing the process to increase its tailorability based on the identified context factors, as well as developing and evaluating a concept for rule-based tailoring workshops.

\subsection{Unit of analysis 2: PDP-level analysis}

$\mathrm{C}-2$ had the same focus in terms of methodology, applying the following methods of information acquisition:

- Comparison of pre-established process variants for a particular context factor (Standard PDP for internal projects vs. PDP variant regarding cooperation projects with strategic partners)

- Observation and documentation of three complete tailoring workshops conducted at the beginning of new projects

- Analysis of milestone approval documents from previous projects

18 context factors were identified and documented along with their impacts as briefs using presentation software. The description is based on the meta model (rules with simple and combined conditions, context factors as conditions, impacts on individual process elements). The context factors were on a high-level, related to project objectives, with a broad range of impacts on different departments across the PDP. One example is the development of product variants within a project: Depending on the situation, whether variants are developed or not, activities regarding variant management can be dropped. If variants are developed, different methods are used, depending on the number of variants, resulting in different activity: For high variety, a matrix-based method to guarantee consistent variants is applied; for smaller numbers of variants (e.g. for emerging markets), simple configuration lists are sufficient. Further context factors are: the part categories which are affected by a project (e.g. new parts developed and manufactured in-house, developed in-house and manufactured externally, developed and manufactured externally, or carry-over with/without modification), whether virtual mock-ups are planned for a project, or whether a project is a collaboration with strategic partners.

According to the assessment of company experts, the identified set of context factors represents a valid "80\%-solution", which is estimated to be sufficient as a first support and merits going ahead with further testing. In their opinion, a $100 \%$ complete solution is neither possible nor required to be of substantial help for tailoring. However, further tailoring needs should be identified within individual departments.

\subsection{Evaluation results and discussion}

Judging from the responses from the semi-structured evaluation interviews $(n=8)$, the presented design support is overall seen positive in terms of its applicability and usefulness. Over the course of C-1, project engineers expressed interest in being able to contribute and be involved in tailoring, since they are directly impacted by the tailored process. The interviews within both case studies indicated, that the tailoring-relevant knowledge is unevenly distributed within the organization, with knowledge carriers (project engineers) required during tailoring but currently often not involved to proactively contribute to tailoring. The application of the methodology in both studies showed that the identification of relevant context factors as well as process impacts was feasible in this setting. Using the meta model, this knowledge has then been documented in the form of tailoring rules in both cases, for communication with and reuse by other process stakeholders. The rules have been seen as valuable input for discussions in future tailoring workshops, in order to increase their efficiency. The defined tailoring rules were evaluated as being useful for systematic decision making, providing rationale on which process elements to focus during tailoring, increasing transparency and traceability of tailoring decisions. According to the interviewees, context factors have to be concrete enough to minimize room for interpretation and enable the definition of valid impacts. The formulation of binary yes/no-variables has been favoured, with discrete, clearly observable values being the minimum requirement. Furthermore, context factors should be static at the time of tailoring going forward. Therefore, different tailoring points have been defined, were specific tailoring rules are applied.

Identifying and formulating the tailoring rules (context factors as well as impacts) is not a trivial task and resource-intensive, requiring input from knowledge carriers. For extensive PDPs, the process should 
be partitioned in order to parallelize the information acquisition. Nonetheless, within the investigated case, valid rules could be defined but need to be tested for reliability in future tailoring workshops.

The documented rules are seen to reduce dependence on individual stakeholders. They support the communication of tailoring decisions, especially with broader implications. They have the potential to avoiding misunderstandings and conflicts between different departments, as several rules had department-spanning impacts. Comparing both case studies, dependencies between rules on different process levels could be identified. Since the developed methodology is intended to be applied iteratively, thereby expanding the TSM, this further motivates the development of a structural analysis framework, with which an automated analysis of rule dependencies can be conducted. The identified context factors differ quite substantially, due to the different perspectives: C-2 has produced context factors on a more general level, and more related to project goals and overall properties. $\mathrm{C}-1$ has produced more productrelated context factors, e.g. related to part geometry. Hence, tailoring decisions are not only highly organization-specific, but also differ within a single organization, requiring a differentiated analysis of individual processes and sub-processes with input from different stakeholder levels.

A detailed process model helped the acquisition phase, to better identify context factors and their effect on the process. However, we also found that direct impacts can be difficult to define, because the underlying process is not yet fit for tailoring: The necessary elements to satisfy the identified tailoring needs can be missing, too condensed (several activities rolled into one), or not detailed enough. This prompted an adaptation of the methodology to include modelling guidelines on how to handle such situations, by providing process adaptation operators. These indirect impacts should be documented nonetheless. Indirect impacts can still provide valuable input for tailoring workshops. For example, in case study C-2 we found the "amount of carry-over-parts" as well as the "degree of modularity" of a particular development object to be relevant to the workshop participants, but impacts could not be traced to specific process elements.

The experts noted that tailoring always requires expert judgment, with automation only seen as a supporting tool, e.g. to visualize the impacts of process tailoring decisions. This insight further motivates the extension of the TSM analysis, to identify tailoring-induced process dependencies between process stakeholders (e.g. roles) as they are affected by tailoring decisions. This can be used to support communication between stakeholders. The tailoring rules serve as documented experience and best practices, but are ultimately applied using expert judgment.

The evaluation as presented only represents a partial, initial evaluation. Aspects such as the tailoring workshop concept and the developed structural analysis framework are subject to further evaluation within the ongoing case studies as well as an accompanying interview study.

\section{Summary, contribution and outlook}

In the paper at hand, we have presented a design support which enables organizations to methodically adapt complex PDPs to project-specific conditions at specific points of the project (e.g. project kick-off and selected gates). The conducted case studies showed that process tailoring is complex and communication-intensive, requiring an appropriate approach. The developed methodology provides operative guidelines and an adaptable selection supporting methods, specifically focusing on the inclusion of relevant process stakeholders through identification of tailoring-induced communication needs based on structural modeling and analysis (cf. research question, Section 2) (Estefan, 2008).

Our work contributes to transferring knowledge regarding process tailoring from software engineering into iPD, where we found limited coverage. On a theoretical level, our approach combines existing work in the area of process tailoring with a systematic analysis of the acquired tailoring knowledge using structural analysis. Furthermore, due to our empirical case studies, our work contributes practical insights into process tailoring in industrial environments. The design support is based on existing approaches as well as requirements for applicability in practice, thereby addressing issues and research gaps associated with existing approaches. Key process stakeholders are included early on in modelling the TSM, as well as in tailoring workshops, supported through the structural analysis (clustering). The expected benefits of a workshop-based approach to satisfy communication needs during process tailoring have been tentatively corroborated in interviews with industry experts in our case studies. A developed workshop concept is currently subject to evaluation. Due to the partial evaluation in industrial 
case studies, the developed design support represents an applicable approach. The presented modelling approach as well as the methodology are extendable e.g. by developing further methods to support individual phases. While the TSM meta model is intended to be used in combination with a formal approach (using e.g. graphs or a relational database), the underlying logic can be used to initially document tailoring rules in a less formalized way, using e.g. spreadsheet or presentation software. The meta model is independent of established process modelling languages and provides a set of attributes for the documentation of context factors. We have focused on activities as the central element type for process tailoring, however, other variance-afflicted types such as artefacts, documents, or support (systems or methods) could be included in the future.

Currently, we are detailing the analysis framework, by selecting and contextualizing structural metrics and implementing algorithms required to automate the analyses. Furthermore, we continue the empirical work, conducting further case studies to evaluate the modelling- and analysis framework, and the developed workshop concept. The presented top-down approach could be further complemented by a bottom-up approach, which specifically focuses on the development of activity modes by determining their effort/benefit ratio regarding their added value for a project (cf. Browning, 2014).

\section{References}

Andreasen, M., Hansen, C. and Cash, P. (2015), Conceptual design: Interpretations, mindset and models, Springer, Cham. https://doi.org/10.1007/978-3-319-19839-2

Badke-Schaub, P. and Frankenberger, E. (2004), Management kritischer Situationen: Produktentwicklung erfolgreich gestalten, Springer, Berlin. https://doi.org/10.1007/978-3-642-18702-5

Bender, B. and Gericke, K. (2016), "Entwicklungsprozesse", In: Lindemann, U. (Ed.), Handbuch Produktentwicklung, Hanser, München, pp. 401-424. https://doi.org/10.3139/9783446445819.014

Blessing, L. and Chakrabarti, A. (2009), DRM, a Design Research Methodology, Springer London. https://doi.org/10.1007/978-1-84882-587-1

Browning, T.R. (2001), "Applying the design structure matrix to system decomposition and integration problems: A review and new directions”, IEEE Transactions on Engineering Management, Vol. 48 No. 3, pp. $292-306$. https://doi.org/10.1109/17.946528

Browning, T.R. (2014), “A Quantitative Framework for Managing Project Value, Risk, and Opportunity”, IEEE Transactions on Engineering Management, Vol. 61 No. 4, pp. 583-598. https://doi.org/10.1109/TEM.2014.2326986

Browning, T.R., Fricke, E. and Negele, H. (2006), "Key concepts in modeling product development processes", Systems Engineering, Vol. 9 No. 2, pp. 104-128. https://doi.org/10.1002/sys.20047

Chucholowski, N. and Lindemann, U. (2015), "An Initial Metamodel to Evaluate Potentials for Graph-based Analyses of Product Development Projects", Proceedings of DMS 2015 / the 17th International Dependency and Structure Modeling Conference, Fort Worth, Texas, November 4-6, 2015, Carl Hanser, Munich, pp. 7788.

Czarnecki, K. and Eisenecker, U. (2005), Generative programming: Methods, tools, and applications, Addison Wesley, Boston.

Estefan, J.A. (2008), Survey of Candidate Model-Based Systems Engineering (MBSE) Methodologies, rev. B, INCOSE MBSE Initiative, Seattle, WA, USA.

Gericke, K., Eckert, C.M. and Wynn, D. (2016), "Towards a framework of choices made during the lifecycles of process models", Proceedings of DESIGN 2016 / the $14^{\text {th }}$ International Design Conference, Dubrovnik, Croatia, May 16-19, 2016, The Design Society, Glasgow, pp. 1275-1284.

Gericke, K., Meißner, M. and Paetzold, K. (2013), "Understanding the context of product development", Proceedings of ICED'13 / the 19th International Conference on Engineering Design Seoul, Korea, August 1922, 2013, The Design Society, Glasgow, pp. 191-200.

Ginsberg, M.P. and Quinn, L.H. (1995), Process Tailoring and the Software Capability Maturity Model: Technical Report CMU/SEI-94-TR-024, Software Engineering Institute, Carnegie Mellon University, Pittsburgh, Pennsylvania. https://doi.org/10.21236/ADA302689

Graviss, M., Sakrani, S. and Mazzuchi, T.A. (2016), “Tailoring a Large Organization's Systems Engineering Process to Meet Project-Specific Needs", Defense Acquisition Research Journal: A Publication of the Defense Acquisition University, Vol. 23 No. 3, pp. 274-297.

Haskins, C. (2011), Systems engineering handbook: A guide for system life cycle processes and activities, International Council of Systems Engineering, San Diego, California.

Helms, B. (2013), Object-Oriented Graph Grammars for Computational Design Synthesis, PhD thesis, Technischen Universität München. 
Henderson-Sellers, B. and Ralyté, J. (2010), "Situational Method Engineering: State-of-the-Art Review”, Journal of Universal Computer Science, Vol. 16 No. 3, pp. 424-478. https://doi.org/10.3217/jucs-016-03-0424

Hollauer, C. and Lindemann, U. (2017), "Design process tailoring: A review and perspective on the literature", Proceedings of ICoRD 2017 / the International Conference on Research into Design, Vol. 1, Guwahati, India, Springer, Singapore, pp. 609-618. https://doi.org/10.1007/978-981-10-3518-0_53

Hollauer, C., Kattner, N. and Lindemann, U. (2016), "Towards a methodology to support the development of flexible company-specific engineering design processes", Proceedings of PICMET '16, Honolulu, Hawaii, September 4-8, 2016, IEEE, pp. 2018-2030. https://doi.org/10.1109/PICMET.2016.7806724

Hurtado Alegria, J.A. (2012), A Meta-process for Defining Adaptable Software Processes, PhD thesis, Departamento de ciencias de la computación, University of Chile.

Hurtado Alegría, J.A., Bastarrica, M.C., Quispe, A. and Ochoa, S.F. (2011), "An MDE approach to software process tailoring", Proceedings of ICSSP '11/ the 2011 International Conference on Software and Systems Process, Waikiki, USA, May 21-22, 2011, ACM, New York, pp. 43-52. https://doi.org/10.1145/1987875.1987885

Kang, D., Song, I.-G., Park, S., Bae, D.-H. and Kim, H.-K. (2008), “A Case Retrieval Method for KnowledgeBased Software Process Tailoring Using Structural Similarity", Proceedings of APSEC '08 / the 15th AsiaPacific Software Engineering Conference, Beijing, China, December 3-5, 2008, IEEE, pp. 51-58. https://doi.org/10.1109/APSEC.2008.15

Kissel, M.P. (2014), Mustererkennung in komplexen Produktportfolios, PhD thesis, Technischen Universität München.

Kreimeyer, M. (2010), A structural measurement system for engineering design processes, $\mathrm{PhD}$ thesis, Technischen Universität München.

Lindemann, U., Maurer, M. and Braun, T. (2009), Structural complexity management: An approach for the field of product design, Springer, Berlin, Heidelberg. https://doi.org/10.1007/978-3-540-87889-6

Markham, S.K. and Lee, H. (2013), "Product Development and Management Association's 2012 Comparative Performance Assessment Study", Journal of Product Innovation Management, Vol. 30 No. 3, pp. 408-429. https://doi.org/10.1111/jpim.12025

Martinez-Ruiz, T., Munch, J., Garcia, F. and Piattini, M. (2012), "Requirements and constructors for tailoring software processes: a systematic literature review”, Software Quality Journal, Vol. 20 No. 1, pp. 229-260. https://doi.org/10.1007/s11219-011-9147-6

Maurer, M.S. (2007), Structural Awareness in Complex Product Design, PhD thesis, Technischen Universität München.

Park, S., Na, H., Park, S. and Sugumaran, V. (2006), “A semi-automated filtering technique for software process tailoring using neural network”, Expert Systems with Applications, Vol. 30 No. 2, pp. 179-189. https://doi.org/10.1016/j.eswa.2005.06.023

Pedreira, O., Piattini, M., Luaces, R.M. and Brisaboa, R.N. (2007), “A systematic review of software process tailoring”, ACM SIGSOFT Software Engineering Notes, Vol. 32 No. 3, pp. 1-6. https://doi.org/10.1145/1241572.1241584

Pillat, R.M., Oliveira, T.C., Alencar, P.S. and Cowan, D.D. (2015), "BPMNt: A BPMN extension for specifying software process tailoring", Information and Software Technology, Vol. 57, pp. 95-115. https://doi.org/10.1016/j.infsof.2014.09.004

Tittmann, P. (2003), Graphentheorie: Eine anwendungsorientierte Einführung; mit zahlreichen Beispielen und 80 Aufgaben, Fachbuchverl, Leipzig im Hanser-Verl, München.

Walden, D., Roedler, G., Forsberg, K., Hamelin, R. and Shortell, T. (2015), Systems engineering handbook: A guide for system life cycle processes and activities, John Wiley and Sons Inc., Hoboken, New Jersey.

Xu, P. (2005), "Knowledge Support in Software Process Tailoring", Proceedings of HICSS '05 / the 38th Annual Hawaii International Conference on System Sciences, Big Island, Hawaii, January 6, 2005, IEEE. https://doi.org/10.1109/HICSS.2005.380

Xu, P. and Ramesh, B. (2008), "Impact of Knowledge Support on the Performance of Software Process Tailoring", Journal of Management Information Systems, Vol. 25 No. 3, pp. 277-314.

Yin, R. (2014), Case study research: Design and methods, SAGE, Los Angeles.

Zakaria, N.A., Suhaimi, I. and Naz'ri Mahrin, M. (2015), "A Proposed Value-Based Software Process Tailoring Framework", Proceedings of MySEC 2015 / the 9th Malaysian Software Engineering Conference, Kuala Lumpur, Malaysia, IEEE, pp. 149-153. https://doi.org/10.1109/MySEC.2015.7475212

Christoph Hollauer, Dipl.-Ing., Wissenschaftlicher Mitarbeiter

Technical University of Munich, Institute of Product Development

Boltzmannstraße 15, 85748 Garching bei München, Germany

Email: christoph.hollauer@pe.mw.tum.de 